\title{
Estrategia de prevención de obesidad en escolares: Efecto de un programa aplicado a sus profesores (2007-2008)
}

\author{
JULIANA KAIN $^{1 \mathrm{a}}$, BÁRBARA LEYTON ${ }^{1 \mathrm{~b}}$, FERNANDO CONCHA ${ }^{1,2, \mathrm{c}}$, \\ GABRIELA SALAZAR ${ }^{1, d}$, LUZ LOBOS ${ }^{\text {le }}$, FERNANDO VIO ${ }^{1}$
}

\section{Effect of counselling school teachers on healthy lifestyle on the impact of a program to reduce childhood obesity}

\begin{abstract}
Background: In 2007, a preventive strategy for childhood obesity, that included food education and an increase in physical activity, was implemented in seven public schools located in Santiago. In four of these schools, a counselling program about healthy lifestyles for teachers was also carried out. Aim: To test if counselling had an effect in the intervention for children. Material and Methods: Anthropometric measures were assessed in children at baseline and after two years of intervention. Teachers were also evaluated with anthropometry and a blood sample was obtained to measure blood glucose and serum lipids. Results: Four hundred twelve children from schools whose teachers had counselling and 237 children from schools whose teachers did not have it were evaluated. Twenty eight teachers with and 19 with no counselling were also assessed. In children, the overall prevalence of obesity decreased from 20.2 to $18.3 \%$ ( $p=0.03$ ). This reduction was only significant among females. $B M I Z$ score decreased significantly in children of both genders. No effect of counselling was observed on weight reduction. Among teachers that received counseling, the prevalence of obesity decreased from 25 to $22.4 \%$, with significant improvements in blood glucose and HDL cholesterol. Conclusions: Counseling directed at teachers did not improve the effect of a program to reduce obesity among school children.
\end{abstract}

(Rev Med Chile 2010; 138: 181-187).

Key words: Child; Life style; Obesity.

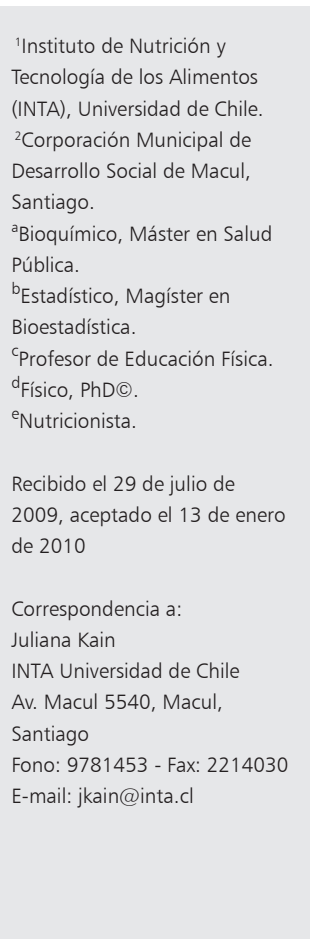

$\mathrm{E}$ n Chile la obesidad ha aumentado significativamente en la población infantil. Es así como datos sobre aproximadamente $77 \%$ de los escolares de primero básico del país revelan que el porcentaje de obesidad ha aumentado de $16 \%$ a $20,8 \%$ entre 2000 y $2008^{1,2}$.

Desde el año 2002, nos hemos enfocado a la prevención de obesidad desde las escuelas básicas públicas, interviniendo durante tres años a escolares de la ciudad de Casablanca ${ }^{3-5}$ y en preescolares y escolares de $1^{\circ}$ a $4^{\circ}$ básico de 7 escuelas de una comuna de Santiago (Macul), del 2006 al 2009. Esta es una comuna de nivel socioeconó- mico medio-bajo y bajo, con una población de aproximadamente 116.000 personas con $13,4 \%$ de pobres (dato 2006). La intervención se realizó en esta comuna por el interés de sus autoridades en prevenir la obesidad, cuya prevalencia en $1^{\circ}$ básico es de alrededor de $20 \%$. Se decidió intervenir a niños más pequeños, ya que es más costo-efectivo cambiar hábitos de vida tempranamente para lograr un impacto duradero ${ }^{6,7}$. Además, en 4 de las 7 escuelas, aparte de aplicar la estrategia dirigida a los escolares, se implementó un programa de apoyo personalizado voluntario a los profesores, Consejería en Vida Sana $(\mathrm{CVS})^{8}$, ya que normal- 
mente a ellos sólo se les utiliza como medio para realizar actividades en alimentación y actividad física con sus alumnos, y no como sujetos de una intervención.

La hipótesis planteada fue que profesores partícipes de un programa tendiente a cambiar sus propios hábitos de vida estarían más motivados en aplicar la estrategia de prevención de obesidad a sus alumnos, y que esto se traduciría en una mayor efectividad de la intervención en los niños.

El propósito de este artículo es mostrar la efectividad de la intervención después de aplicarla durante 2 años en todos los escolares y además compararla en escolares cuyos profesores tuvieron Consejería (PCC) versus aquellos niños cuyos profesores no tuvieron (PSC).

\section{Metodología}

En la comuna de Macul hay 7 escuelas básicas públicas. En 2006 se aplicó un proyecto piloto durante 6 meses a una muestra de 522 alumnos de prekinder a $4^{\circ}$ básico con el fin de determinar la factibilidad de implementar esta estrategia en todos los niños. Se observó una tendencia de los escolares de menor edad de disminuir la obesidad, mientras que en los profesores, disminuyó levemente el IMC y la circunferencia de cintura (CC); sin embargo, sus parámetros bioquímicos no variaron?.

A continuación se describen los principales aspectos de la intervención aplicada el 2007 y 2008.

\section{Diseño del estudio}

Estudio no aleatorio en el cual se intervinieron durante 2 años a los alumnos de prekinder a $3^{\circ}$ básico de las 7 escuelas básicas municipalizadas de Macul. Adicionalmente, en 4 escuelas (seleccionados por las autoridades educacionales) se intervino a los profesores de esos niveles con el programa que incluyó la CVS, evaluación antropométrica y de algunos parámetros bioquímicos. Los resultados se presentan como comparación final e inicial de: a) porcentaje de obesidad y puntaje $Z$ de IMC de todos los niños y b) las variables anteriores entre los 2 grupos de escolares, según si sus profesores siguieron o no el programa. Fueron excluidos del análisis por grupo, los niños de un curso cuyo profesor no quiso participar en la consejería $(n=22)$ y los niños de 3 cursos cuyos profesores no cuentan con exámenes bioquímicos al inicio o al final $(\mathrm{n}=$ 70). Se compararon además las mediciones antropométricas de los PCC y las efectuadas en el resto de los profesores; sin embargo, las determinaciones bioquímicas sólo fueron comparadas en los PCC, ya que no se contó con autorización de medirlas en los otros.

\section{Sujetos}

a) Preescolares y escolares

Participaron en este estudio todos los preescolares y escolares hasta $3^{\circ}$ básico (en el 2007) de los 7 colegios durante 2 años. El $\mathrm{N}$ inicial de niños fue de 942. La efectividad de la intervención se midió comparando los resultados finales con los iniciales de las variables antropométricas, de acuerdo al criterio de Habicht y cols ${ }^{10}$, para medir impacto en intervenciones en nutrición. El $\mathrm{N}$ de niños que cuenta con mediciones completas durante 2007 y 2008 fue de 741 niños. Esta pérdida, que es considerable (22\%), se debió básicamente a cambio de colegio por traslado de sus padres (16\%), y el resto, por no estar presente los niños en los días de medición. Vale la pena comentar que este porcentaje de pérdida es muy similar al observado en las escuelas municipales de esta comuna. No hubo diferencias en el estado nutricional inicial de los niños que están incluidos en la comparación con el de aquellos no presentes al final. En la comparación por grupo, el $\mathrm{N}$ total fue de 649 niños, 412 niños con PCC y 237 niños con PSC (Tabla 1 muestra la distribución de los niños en muestra total, por grupo y sexo).

\section{b) Profesores}

El número de PCC fue 26 ( 7 educadoras de párvulos y 19 profesores de $1^{\circ}$ a $3^{\circ}$ básico en el 2007). Los PSC sumaron 19, de los cuales 4 eran educadoras de párvulos.

El proyecto fue aprobado por el Comité de Ética del INTA y cada profesor intervenido debió firmar un consentimiento para participar en el estudio. Como la intervención dirigida a los niños fue curricular, los investigadores junto a las autoridades educacionales estimaron que no era necesario obtener consentimiento de ellos.

\section{Intervención}

\section{Escolares}

a) Componente Alimentación/Nutrición

Preescolares: se entregó a las educadoras, el 
material ya validado "Educación en Nutrición para la Educación Preescolar"11.

Escolares de $1^{\circ}$ y $2^{\circ}$ básico: se entregó un material educativo desarrollado en el INTA sobre alimentación saludable ${ }^{12}$.

Escolares de $3^{\circ}$ y $4^{\circ}$ básico: se entregó a los profesores y alumnos el material validado "Educación en Nutrición para Escolares de Educación Básica"13.

Capacitación de Educadoras de Párvulos y Profesores: Se capacitó en el uso de los materiales mencionados anteriormente y formas de evaluar el aprendizaje de los niños. Durante 2007, esta capacitación consistió en dos sesiones de 90 minutos cada una del grupo completo, más 3 sesiones de 20 minutos por escuela, mientras que en 2008 , se realizó una sesión del grupo completo y supervisión de la aplicación de las actividades por la nutricionista.

\section{b) Componente Actividad Física}

Preescolares: se entregó a las educadoras el material ya validado "Educación en Actividad Física para la Educación Preescolar"14. En las 2 sesiones grupales, mencionadas anteriormente, las educadoras fueron capacitadas en el uso de este material.

Escolares: en 2007, los docentes fueron capacitados durante 2 meses (en tres oportunidades) por profesores de educación física con el objetivo de mejorar la calidad de las clases de educación física, utilizando 9 fichas que incluyen 20 actividades prácticas cada una. En 2008, todas las clases de educación física fueron efectuadas por profesores de esa disciplina. Tanto en 2007 como en 2008, se realizaron 4 sesiones de clases en dos días distintos.

Implementos deportivos: se entregaron en todos los colegios.

\section{Profesores}

Las educadoras de párvulos y docentes de $1^{\circ} \mathrm{a}$ $3^{\circ}$ básico de 4 escuelas, además de ser partícipes de las actividades mencionadas anteriormente, fueron apoyados con el CVS 3 veces durante cada año y se les determinó IMC, CC y algunos parámetros bioquímicos, 2 veces cada año. La CVS es una acción que se incorpora a las actividades realizadas por el personal de salud para detectar factores de riesgo (estilos de vida no saludables), valorando aquellos que son saludables, aconsejando cambios de conducta ${ }^{8}$. En cada sesión la nutricionista registró la frecuencia de consumo de alimentos críticos, frecuencia y tiempo de actividad física semanal. Los profesores fueron pesados y medidos con poca ropa y sin zapatos con balanzas digitales marca Pesatronic EFIP-1803030-21 con tallímetro incorporado (precisión es de $50 \mathrm{~g}$ y $0,1 \mathrm{~cm}$ ). La CC se evaluó con una cinta inelástica marca SECA. Una auxiliar de enfermería les extrajo sangre en condiciones de ayuno; las determinaciones fueron realizadas por un laboratorio externo.

\section{Variables}

En los niños se calculó la variación del porcentaje de obesidad y el $\mathrm{z}$ de IMC al final del segundo año. En los profesores, las variables evaluadas fueron: IMC, CC, glicemia, colesterol total, HDL, LDL y triglicéridos y en los PCC, el grado de cumplimiento de metas en los aspectos de alimentación $\mathrm{y}$ actividad física.

El peso y la talla fueron determinados por dos nutricionistas entrenadas y estandarizadas. Se calculó el IMC $\left(\mathrm{kg} / \mathrm{m}^{2}\right)$, clasificando a los niños como obesos si su IMC era $>2$ DE de la Referencia OMS 2006 y $2007^{15,16}$; posteriormente se calculó el z IMC. Por cada profesor se contó con determinaciones de IMC y CC; adicionalmente para los 26 PCC se obtuvieron mediciones de glicemia, colesterol total, HDL LDL y triglicéridos.

\section{Análisis Estadístico \\ Escolares}

En los niños, la efectividad de la estrategia de prevención de obesidad se determinó comparando tanto la variación del porcentaje de obesidad como la del z IMC. Este análisis se efectuó en la muestra total/sexo y grupo/sexo, es decir en niños separados en dos grupos, aquellos con PCC y con PSC. Se creó una nueva variable para visualizar el cambio de la proporción de obesidad en el tiempo, donde el valor negativo significa que un sujeto pasa de no obeso a obeso; el valor cero, que no hubo cambio y el valor positivo, que el sujeto pasa de obeso a no obeso. Se determinó si había diferencias entre grupos de esta nueva variable utilizando el test no paramétrico de Wilcoxon para muestras independientes, en cambio para verificar si hubo diferencia significativa en la muestra total y según sexo, se utilizó el test anterior, pero para muestras pareadas.

Para determinar si el cambio de z IMC para la muestra total, por sexo y por grupo fue significativo, se usaron modelos de covarianza Proc Mixed 
que controla por el valor inicial y prueba la interacción grupo * tiempo. Se consideró significativo si el p era $<0,05$ (Proc Mixed SAS versión 9.1.3).

\section{Profesores}

Se determinó el cambio de las medidas antropométricas comparando aquellos con y sin consejería, usando el test $t$, mientras que para los parámetros bioquímicos se utilizó el test de Wilcoxon para muestras pareadas. Se comparó el grado de cumplimiento de las metas planteadas para cada alimento, frecuencia y tiempo total de actividad física semanal con el test de Wilcoxon para muestras pareadas.

\section{Resultados}

La Tabla 1 muestra los resultados iniciales y finales del porcentaje de obesidad según sexo en muestra total y por grupo. El porcentaje de obesidad en la muestra total disminuyó significativamente de $20,2 \%$ a $18,3 \%(p=0,031)$. Esta disminución se observó sólo en las niñas. Según grupo, este porcentaje disminuyó levemente $(0,8$ $\%$ ) en los escolares cuyos profesores fueron intervenidos, debido al descenso observado en las niñas, mientras que en los escolares cuyos profesores no tuvieron consejería, la disminución fue significativa $(4,2 \%)$. En este caso, el descenso se observa en ambos sexos, pero fue sólo significativo en las niñas $(\mathrm{p}=0,01)$.
La Tabla 2 muestra los valores iniciales y finales del z IMC en la muestra total de escolares intervenidos, por grupo y sexo. El z IMC disminuyó significativamente ( $\mathrm{p}<0,0001)$, en la muestra total y por sexo, sin embargo, no se observó diferencia por grupo (el p de la interacción grupo ${ }^{\star}$ tiempo fue 0,08$)$. En la comparación por grupo y sexo, se observa una disminución significativa sólo en aquellos varones cuyos profesores no fueron intervenidos $(\mathrm{p}=0,0037)$.

La Tabla 3 muestra que aun cuando en los PCC el promedio de IMC no varió, el porcentaje de obesidad disminuyó no significativamente (de $25 \%$ a $22,4 \%$ ). Al comparar los cambios con el grupo de profesores sin consejería, se observó que estos, en promedio, aumentaron no significativamente la CC y el porcentaje de obesidad. Respecto a los cambios en los parámetros bioquímicos medidos sólo en los profesores intervenidos (Tabla 4), se observó una mejoría general de estos, sin embargo, la variación fue sólo significativa para glicemia y colesterol HDL.

Respecto al grado de cumplimiento de las metas planteadas por los mismos profesores, los resultados fueron los siguientes: $67 \%$ de los profesores cumplió con aumentar el tiempo destinado a actividad física, $61 \%$ y $68 \%$ respectivamente, con aumentar el consumo de frutas y verduras, mientras que $85 \%$ disminuyó el consumo de pan. Sólo se constataron diferencias significativas entre valor de la meta y el inicial para tiempo de actividad física y consumo de pan.

Tabla 1. Número de Escolares Intervenidos y Variación del Porcentaje de obesidad en muestra total, por grupo y sexo. 2007- 2008

\begin{tabular}{|lccc|}
\hline & H & M & Total \\
Muestra Total & $\mathbf{n}=\mathbf{3 9 0}$ & $\mathbf{n}=\mathbf{3 5 1}$ & $\mathbf{n}=\mathbf{7 1 4}$ \\
\% obesidad inicial & 21,5 & 18,8 & 20,2 \\
\% obesidad final & 21,0 & $15,4^{*}$ & $18,3^{*}$ \\
Niños cuyos Profesores tuvieron Consejería & $\mathbf{n}=\mathbf{2 0 9}$ & $\mathbf{n}=\mathbf{2 0 3}$ & $\mathbf{n}=\mathbf{4 1 2}$ \\
\% obesidad inicial & 21,9 & 18,1 & 20,0 \\
\% obesidad final & 22,7 & $15,7^{*}$ & 19,2 \\
Niños cuyos Profesores no tuvieron Consejería & $\mathbf{n = 1 3 5}$ & $\mathbf{n = 1 0 2}$ & $\mathbf{n = 2 3 7}$ \\
\% obesidad inicial & 20,7 & 20,6 & 20,7 \\
\% obesidad final & 17,8 & $14,7^{*}$ & $16,5^{*}$ \\
\hline
\end{tabular}

*diferencia significativa entre datos finales e iniciales dentro del mismo grupo (test de Wilcoxon) 
Tabla 2. Variación del puntaje Z de IMC en Escolares Intervenidos en muestra total, por grupo y sexo (2007- 2008)

\begin{tabular}{|c|c|c|c|}
\hline & $\mathbf{H}$ & M & Total \\
\hline Muestra Total & Prom (DE) & Prom (DE) & Prom (DE) \\
\hline ZIMC inicial & $1,07(1,2)$ & $0,98(1,1)$ & $1,03(1,1)$ \\
\hline ZIMC final & $0,94(1,1)^{\circ}$ & $0,90(1,0)^{\circ}$ & $0,92(1)^{\circ}$ \\
\hline \multicolumn{4}{|c|}{ Niños cuyos Profesores tuvieron Consejería } \\
\hline ZIMC inicial & $1,1(1,2)$ & $0,96(1,0)$ & $1,03(1,1)$ \\
\hline ZIMC final & $1,0(1,1)$ & $0,87(1,0)$ & $0,94(1,0)$ \\
\hline \multicolumn{4}{|c|}{ Niños cuyos Profesores no tuvieron Consejería } \\
\hline ZIMC inicial & $1,03(1,1)$ & $1,04(1,1)$ & $1,03(1,1)$ \\
\hline ZIMC final & $0,81(0,94)^{\circ}$ & $0,98(1,0)$ & $0,9 \quad(1,0)$ \\
\hline
\end{tabular}

'diferencia significativa entre datos finales e iniciales en muestra total (Proc Mixed SAS 9.1.3). ${ }^{\circ}$ diferencia significativa entre datos finales e iniciales dentro de cada sexo por grupo (Proc Mixed SAS 9.1.3).

Tabla 3. Variación del Estado Nutricional de Profesores con y sin consejería durante 2 años (2007-2008)

\begin{tabular}{|c|c|c|c|c|}
\hline \multirow[b]{2}{*}{ Antropometría } & \multicolumn{2}{|c|}{ Mayo 2007} & \multicolumn{2}{|c|}{ Noviembre 2008} \\
\hline & $\begin{array}{l}\text { Prof con } \\
\text { Consejería } \\
(n=28)\end{array}$ & $\begin{array}{c}\text { Prof sin } \\
\text { Consejería } \\
(n=19)\end{array}$ & $\begin{array}{l}\text { Prof con } \\
\text { Consejería } \\
(n=28)\end{array}$ & $\begin{array}{c}\text { Prof sin } \\
\text { Consejería } \\
(n=19)\end{array}$ \\
\hline IMC & $27,1 \quad(3,7)$ & $27,2(3,9)$ & $26,8(3,7)$ & $26,8(3,9)$ \\
\hline Circunferencia de Cintura (cm) & $84,7(10,4)$ & $84,3(10,5)$ & $84,9(7,9)$ & $86,7(10,9)$ \\
\hline$\%$ Obesidad & 25,0 & 21,4 & 22,4 & 24,6 \\
\hline \% Sobrepeso & 39,3 & 42,9 & 42,9 & 35,7 \\
\hline
\end{tabular}

Tabla 4. Variación de parámetros bioquímicos de profesores con Consejería durante 2 años (2007-2008)

\begin{tabular}{|c|c|c|}
\hline $\begin{array}{c}\text { Parámetros } \\
\text { Bioquímicos } \\
n=26\end{array}$ & $\begin{array}{c}\text { Mayo } \\
2007 \\
\text { Prom (DE) }\end{array}$ & $\begin{array}{l}\text { Noviembre } \\
2008 \\
\text { Prom (DE) }\end{array}$ \\
\hline Glucosa (mg/dl & $93,3 \quad(7,6)$ & $88,0 \quad(6,2)^{*}$ \\
\hline Colesterol total (mg/dl) & $201,8(42,5)$ & $201,2(40,4)$ \\
\hline Colesterol HDL (mg/dl) & $48,1 \quad(9,9)$ & $57,4(11,6)^{*}$ \\
\hline Colesterol LDL (mg/dl) & $129,9(33,2)$ & $118,3(39,2)$ \\
\hline Triglicéridos (mg/dl) & $118,6(65,0)$ & $107,2(53,9)$ \\
\hline
\end{tabular}

*diferencia significativa (Test de Wilcoxon).

\section{Discusión}

En este estudio se ratifica que la prevalencia de obesidad en preescolares y escolares chilenos es muy alta. Los resultados más importantes muestran que la estrategia fue efectiva, medida tanto a través del cambio del porcentaje de obesidad como del z IMC, sin embargo, la hipótesis planteada no se cumplió, ya que en los niños cuyos profesores tuvieron consejería, se observaron los menores cambios. Vale la pena comentar cómo en la comparación de resultados de la intervención por grupo, éstos son distintos si se usa como variable respuesta el porcentaje de obesidad o el z IMC. Con la primera variable, sólo se observa 
una diferencia significativa en las niñas de ambos grupos, mientras que con z IMC, la efectividad fue significativamente mayor en los hombres cuyos profesores no fueron intervenidos. Esto se debe a que en el primer caso se mide lo que ocurre en el extremo de la curva de distribución (obesos); en cambio, el z IMC da cuenta de cómo cambia el grupo completo. En este caso, la curva se desplazó hacia la izquierda, ya que el promedio de z IMC disminuyó. Ambos cambios son deseables, ya que por un lado se espera que el número de niños obesos disminuya, o sea que pasen a ser no obesos, y por otro lado que el IMC promedio del grupo completo también disminuya, En este estudio, más niñas obesas pasaron a no obesas. Es interesante constatar que en los escolares de Casablanca la mayor disminución de obesidad también fue observada en las niñas ${ }^{3}$. Pensamos que esto podría deberse en parte a que en las niñas se observaron mejorías más importantes en las clases de educación física (datos no mostrados).

La efectividad de esta intervención fue mucho menor que la obtenida en escolares de Casablan$\mathrm{ca}^{4,5}$, donde la prevalencia de obesidad al final de 2 años de intervención se redujo prácticamente a la mitad. Pensamos que estas diferencias podrían deberse en parte a que en colegios de ciudades pequeñas es más factible apoyar y supervisar la implementación de actividades. En Casablanca se permitía que la nutricionista del proyecto evaluara en las salas el cumplimiento de las actividades. Además se asignó más tiempo para capacitar a los profesores. La participación de los padres, aspecto clave en lograr cambio de hábitos en sus hijos ${ }^{17}$, se logró en Casablanca, mientras que en Macul, ésta fue mínima. Esto no sorprende, ya que es sabido que la asistencia de apoderados es alta en ciudades pequeñas y baja en las más grandes, especialmente en las escuelas públicas.

Los profesores tienen en promedio un IMC alto. Aún cuando el promedio de IMC no varió, en los PCC, la glicemia y colesterol HDL mejoraron. Esto podría atribuirse a los cambios alimentarios y de actividad física. Es necesario reconocer que la muestra de profesores fue muy pequeña, por lo que no es posible extrapolar estos resultados a un universo mayor. Lo que si podemos informar es que los profesores manifestaron estar muy conformes por la orientación hacia su salud otorgada por un profesional.

La literatura muestra cada vez más que en las estrategias de prevención de obesidad a nivel escolar los profesores son claves ${ }^{18,19}$. Conviene, sin embargo, determinar previamente si están dispuestos a asumir ese rol$^{20}$. Existen pocas publicaciones sobre intervenciones que den atención personalizada a los profesores Hay un estudio parecido al nuestro realizado en Estados Unidos de Norteamérica ${ }^{19}$ cuyo objetivo fue determinar si un programa de salud aplicado a profesores lograba impactar la intensidad de entrega del currículo a los escolares y obtener mejores resultados cognitivos, conductuales y fisiológicos en los profesores participantes. En este caso, los colegios controles también recibieron un programa básico cuyo objetivo fue que los escolares aumentaran el consumo de frutas y verduras. No se encontró evidencia de que este programa modificase favorablemente el consumo de frutas y verduras en los niños, ni que los profesores intervenidos obtuvieran mejores resultados en sus determinaciones ${ }^{21}$.

Este estudio tiene varias limitaciones, siendo la más importante el no contar con un grupo control. Aún cuando los resultados no pueden generalizarse a otras poblaciones, fue posible determinar que esta intervención fue efectiva. Además se constató en la pequeña muestra de profesores que la CVS fue bien acogida y produjo resultados positivos en su salud, sin embargo, no tuvo efecto en la obesidad de los niños. Aún con las limitaciones descritas, es probable que aplicando esta estrategia en escuelas públicas de características similares de Santiago, no se obtengan mejores resultados, debido al sistema educacional existente, que incluye pocas posibilidades de capacitación docente, baja motivación de los profesores y pérdida de clases por distintos motivos.

Agradecimientos: Los autores desean agradecer a la Empresa Tresmontes-Lucchetti y la Corporación Municipal de Desarrollo Social de Macul por el apoyo brindado para llevar a cabo este estudio.

\section{Referencias}

1. www.junaeb.cl [Consultado en julio 2009].

2. Bulletin of the World Health Organization. Use and interpretation of anthropometric indicators of nutritional status. WHO, Geneva, 1986.

3. Kain J, Vio F, Leyton B, Cerda R, Olivares S, Uauy R, et al. Estrategia de promoción de la salud de escolares 
de educación básica municipalizada de la comuna de Casablanca, Chile. Rev Chil Nutr 2005; 32: 126-32.

4. Kain J, Uauy R, Leyton B, Cerda R, Olivares S, Vio F. Efectividad de una intervención en educación alimentaria y actividad física para prevenir obesidad en escolares de la ciudad de Casablanca, Chile (2003-2004). Rev Méd Chile 2008; 136: 22-30.

5. Kain J, Leyton B, Cerda R, Vio F, Uauy R. Two-year controlled effectiveness trial of a school-based intervention to prevent obesity in Chilean children. Public Health Nutr 2008; 23: 1-11.

6. Dietz W, Robinson T. Overweight Children and Adolescents. NEJM 2005; 352: 2100-9.

7. Kipping R, Jago R, Lawlor D. Obesity in children: prevention and management. BMJ 2008; 327: a1848.

8. INTA/MINSAL. www.inta.cl/materialEducativo/Consejeriasenvidasana.pdf [Consultado en abril 2009].

9. Kain J, Concha F, Salazar G, Leyton B, Rodríguez MP, Zeballos X, Vio F. Prevención de Obesidad en Preescolares y Escolares de Escuelas Municipales de una Comuna de Santiago de Chile: proyecto piloto 2006. ALAN 2009; 59: 139-46.

10. Habicht JP, Victora C, Vaughan J. Evaluation designs for adequacy, plausability and probability of public health programme: performance and impact. Int J Epidemiol 1999; 28: 10-8.

11. Guía y Manual de Alimentación para la Educación Parvularia. Eds. M Andrade y J Rojas. Elaborado como parte del Proyecto Intervención Comunitaria en Nutrición y Actividad Física en Párvulos. Santiago, 2006.

12. Andrade M. Educación en alimentación y nutrición para el Nivel Básico $1^{\circ}$ y $2^{\circ}$. Guía Didáctica para el Profesor y Guía Didáctica para el Alumno. Mayo 2007.

13. Olivares S, Morón C, Kain J, Zacarías I, Andrade M, Lera L, Díaz N, Vio F. Propuesta metodológica para incorporar la educación en nutrición en la enseñanza básica: la experiencia chilena. ALAN 2004; 54 (Supl1): 33-9.

14. Guía y Manual de Actividad Física para la Educación Parvularia. Ed. F Concha. Elaborado como parte del Proyecto Intervención Comunitaria en Nutrición y Actividad Física en Párvulos. Santiago, 2006.

15. www.who.int/childgrowth/en [Consultado en noviembre 2008].

16. www.who.int/growthref/en [Consultado en noviembre 2008].

17. Nicklas TA, Hayes D; American Dietetic Association. Position of the American Dietetic Association: nutrition guidance for healthy children ages 2 to 11 years. JADA 2008; 108: 1038-44.

18. Story M, Kaphingst K, French S. The role of schools in obesity prevention. Future Child 2006; 16: 10942.

19. Spiegel S, Foulk D. Reducing overweight through a multidisciplinary school-based intervention. Obesity 2008; 14: 86-96.

20. Yager Z, O'Dea J. The role of teachers and other educators in the prevention of eating disorders and child obesity: what are the issues? Eat Disord 2005; 13: 261-78.

21. Resnicow K, Davis M, Smith M, Baranowski T, Lin LS, Baranowski J, Doyle C, Wang D. Results of the TeachWell Worksite Wellness Program. Am J Public Health 1998; 88: 250-7. 